\title{
Current Index: A Proposal for a Dynamic Rating System for Researchers
}

\section{Dalibor Fiala}

University of West Bohemia, Department of Computer Science and Engineering Univerzitní 8, 30614 Plzeň, Czech Republic

Phone: +420 3776324 29, fax: +420 3776324 02, email: dalfia@kiv.zcu.cz

\begin{abstract}
An index is proposed that is based on the h-index and a 3-year publication/citation window. When updated regularly, it shows the current scientific performance of researchers rather than their life-time achievement as indicated by common scientometric indicators. In this respect, the new rating scheme resembles established sports ratings such as in chess or tennis. By the example of ACM SIGMOD E. F. Codd Innovations Award winners and Priestley Medal recipients, we illustrate how the new rating can be represented by a single number and visualized.
\end{abstract}

Keywords: Rating, ranking, h-index, citations, publications, time.

\section{Introduction}

Hirsch proposed the h-index that combined both the productivity and impact of an individual researcher in a single number (Hirsch, 2005). The index is defined as follows: if we have a set of publications ordered by the number of times they are cited in descending order, the index $h$ is the largest number $h$ such that there are $h$ publications having at least $h$ citations each. Thus, a scholar with an h-index of 20 has published 20 papers at least (productivity) and has received no less than 400 citations (impact). The h-index attained a great popularity and was mathematically analyzed and praised, but it was also soon discovered that various corrections were needed. For instance, the h-indices of two researchers from different research fields or subfields are incomparable because publication and citation practice may vary to a great extent between those two fields. Also, it would be unfair to consider the h-indices of two scientists the same if one of the researchers always publishes with a large group of co-authors and the other researcher only publishes alone. In addition, author self-citations can inflate the h-index, etc. To remedy this situation, many h-index variants have been proposed, but their description is not the concern of this short paper that does not aim at the shortcomings above. This is a preprint of an article accepted for publication in Journal of the American Society for Information Science and Technology copyright (C) 2013 (American Society for Information Science and Technology). 
The h-index and other metrics based on it can be applied to any set of publications, for instance aggregated by institutions, countries, or journals, but this paper deals with individual researchers.

Scientometric indicators such as citation counts or h-index generally play in favour of more senior researchers because these simply have had more time to publish and collect citations. Therefore, the current metrics indicate a kind of lifetime achievement instead of current (most recent) performance, which is reflected in many sports ratings. There is a great need for an "age normalization" factor to be able to fairly compare researchers of different ages. Also, the new indicator should be able to grow as well as decline - it should be dynamic. We will introduce a dynamic indicator of scientific performance that will not only increase in time but also decrease according to the current publication activity and citation reputation. A model of such an indicator can be the h-index ( $\mathrm{h}$ bar), which, contrary to the hindex, can decrease in time (Hirsch, 2010). But a decrease can only occur if the researcher under examination publishes new articles. If he/she stops publishing, the h-index (as well as all other related metrics) will never decline - it can only remain the same or grow. Our "Current Index" is able to change over time (increase as well as decrease) even if the scientist under study is not active because the new indicator considers a 3-year time window for both publications and citations and, therefore, reflects current performance rather than life-time achievement. This feature is common in many established sports rating systems such as in chess (FIDE Ratings, ratings.fide.com) or tennis ${ }^{1}$, where the rating scheme is not biased towards more senior players. But we must be cautious with the Current Index as a researcher's performance is not always quantitatively countable and clear-cut compared to an athlete's performance. Therefore, whether the proposed scheme is a good "rating" mechanism for the evaluation of researchers needs to be debated.

\section{Methods and data}

In October 2012 we collected publication and citation data of all twenty ACM SIGMOD Edgar F. Codd Innovations Award ${ }^{2}$ winners from Scopus. The Codd Award has been awarded annually since 1992 for outstanding contributions in the field of databases. We wanted to determine the winners' Current Index in the years 2003 - 2012. As Current Index (CI) uses a 3 -year publication/citation window, the actual data collection time span was $2000-2011$. For

\footnotetext{
${ }^{1}$ ATP Rankings, http://www.atpworldtour.com/Rankings/Singles.aspx

${ }^{2}$ Codd Award, http://www.sigmod.org/sigmod-awards/sigmod-awards\#innovations
}

This is a preprint of an article accepted for publication in Journal of the American Society for Information Science and Technology copyright (C) 2013 (American Society for Information Science and Technology). 
instance, the 2012 rating of a researcher is based on the papers published by him/her in the period 2009 - 2011 and on the citations received by these papers in the same period. Similarly, the 2003 rating takes into account the articles published in $2000-2002$ and the citations to these articles in 2000 - 2002. For the sake of simplicity, we considered all document types and did not discard self-citations. Table 1 shows the results of our data collection for Héctor García-Molina, who won the Codd Award in 1999. In the rating year 2003, he published 29 publications that were cited 24 times, thus producing an h-index of 3 (denoted as h3-index in Table 1). Then, regarding the h3-index and the citation count, his $C I(2003)$ is $3_{24}$. It is a single compound number consisting of the h3-index and the citation count as its subscript. By analogy, the ratings of García-Molina are $4_{43}$ in 2004, $7_{140}$ in 2005, and so on. ${ }^{3}$ The interpretation may be that from a modest starting point in 2003 he quickly reached his top form in 2005 and then gradually worsened his performance with a low in 2010 to finally achieve a good shape in 2011 and 2012 again. So far, the number of publications has not been involved because it does not seem practical to integrate it (perhaps as a superscript) in the rating score. Instead, it will be kept separately and used only as a further criterion to differentiate between researchers whose rating is the same. All in all, regularly updated (possibly on a yearly basis) h3-indices and citation counts (together as Current Index) and publication numbers (as a tiebreak score) represent a dynamic rating system changing in time that ranks researchers in a scientific discipline based on their current impact. The yearly ranks of García-Molina in the small set of twenty Codd Award winners are shown in the sixth column of Table 1. In the very last column of Table 1, the standard h-index known at the time of each specific rating year appears as "career h-index". For example, in 2003 the career hindex is based on publications and citations before 2003 (in Scopus), in 2004 it is based on publications and citations before 2004, etc. As we can see, unlike the h3-index that can grow and decline, the "career h-index" is non-decreasing since it represents life-time achievement. It does definitely not reflect current performance.

\footnotetext{
${ }^{3}$ If needed, the ratings can indeed be stored and manipulated as single decimal numbers with a fixed number of decimal digits, e.g. 3.0024, 4.0043, or 7.0140.

This is a preprint of an article accepted for publication in Journal of the American Society for Information Science and Technology copyright (C) 2013 (American Society for Information Science and Technology).
} 
TABLE 1. Personal rating record of Héctor García-Molina.

\begin{tabular}{rcrrrrr}
\hline Year & Rating & h3-index & Citations & Publications & Rank & Career h-index \\
\hline 2003 & $3_{24}$ & 3 & 24 & 29 & 3 & 16 \\
2004 & $4_{43}$ & 4 & 43 & 42 & 2 & 19 \\
2005 & $7_{140}$ & 7 & 140 & 51 & 1 & 22 \\
2006 & $6_{146}$ & 6 & 146 & 53 & 3 & 26 \\
2007 & $5_{82}$ & 5 & 82 & 39 & 3 & 29 \\
2008 & $5_{72}$ & 5 & 72 & 28 & 7 & 32 \\
2009 & $5_{86}$ & 5 & 86 & 27 & 2 & 35 \\
2010 & $4_{103}$ & 4 & 103 & 34 & 3 & 37 \\
2011 & $6_{196}$ & 6 & 196 & 35 & 1 & 40 \\
2012 & $6_{167}$ & 6 & 167 & 30 & 1 & 43 \\
\hline
\end{tabular}

\section{Results and discussion}

Once the rating score has been defined, it can be visualized. Figure 1 displays the rating progress charts of García-Molina and three other arbitrarily chosen Codd Award winners Jeffrey D. Ullman (awarded in 2006), Serge Abiteboul (1998), and Rakesh Agrawal (2000). The blue line represents the h3-index progress and the red vertical bars the citation count achieved in the specific rating year with the actual number displayed next to them. One can immediately grasp when the researchers were "in good shape" (using the sports terminology) and when they were not. The size of the citation bars is proportional to the largest citation count in each researcher's chart and rescaled for a comfort look. Also, the upper bounds of each chart's Y-axis vary so as to visualize the progress of each scientist as well as possible. On the other hand, if the researchers should be compared to one another, the upper bounds of $\mathrm{Y}$-axes and the sizes and scaling factors of citation bars may be adjusted accordingly for an immediate comparison. Note that publication counts are not present in the charts. They could possibly be displayed as bars below the h3-index line opposite the citation bars (which would then have to be depicted above the line only), but this would probably clutter up the charts with too much information.

This is a preprint of an article accepted for publication in Journal of the American Society for Information Science and Technology copyright (C) 2013 (American Society for Information Science and Technology). 

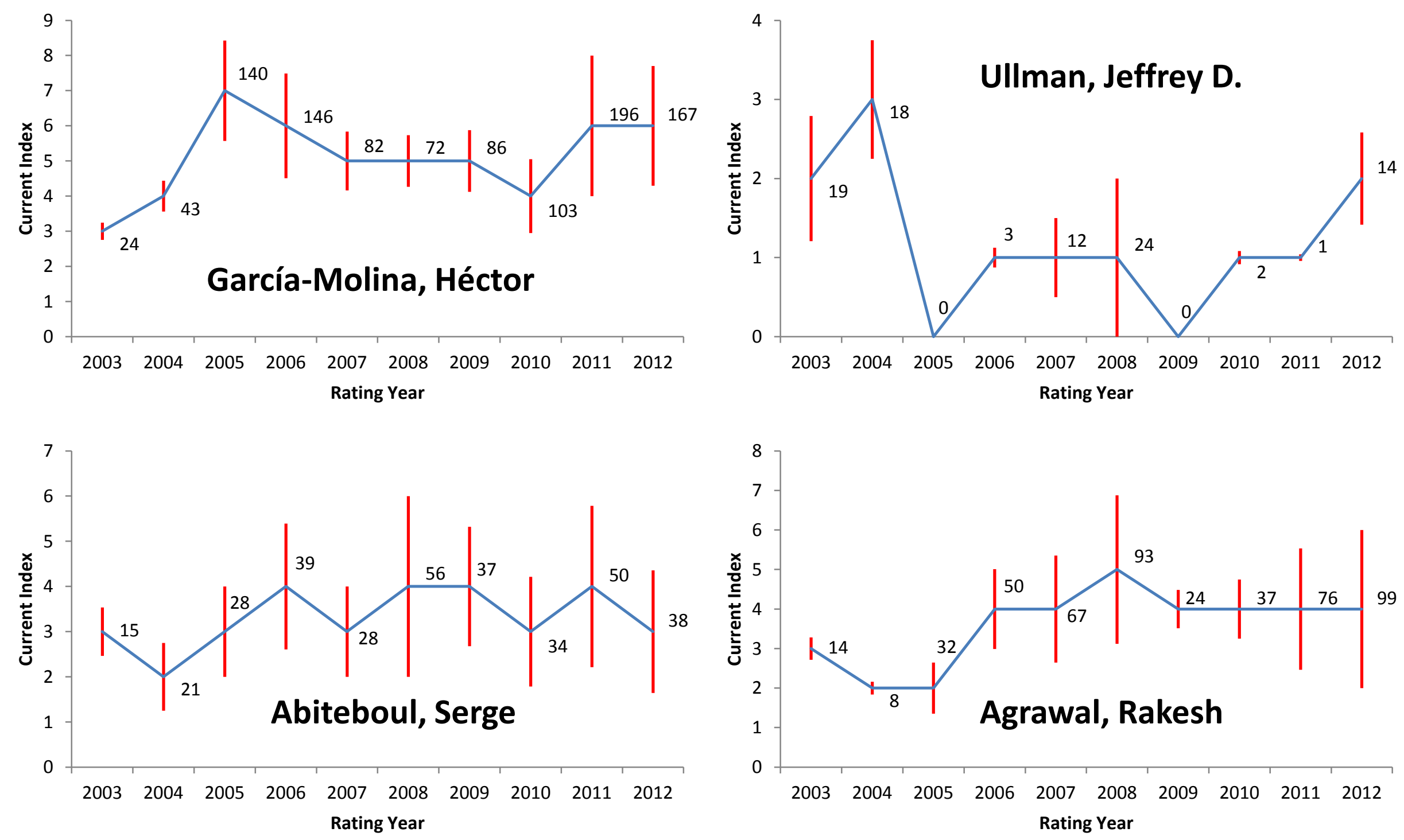

FIG. 1. Rating progress charts of four arbitrary Codd Award winners.

This is a preprint of an article accepted for publication in Journal of the American Society for Information Science and Technology copyright (C) 2013 (American Society for Information Science and Technology). 
If the researchers' ratings in a scientific domain under study are updated once a year, an annual rating table may look like Table 2, in which researchers' 2012 ratings, ranks, and score changes in contrast to the previous year 2011 are shown. The look of the table is somewhat inspired by Live Chess Ratings (www.2700chess.com) in that positive changes (ranking or rating increase) in comparison to the previous rating year are marked in green with " $\uparrow$ " and “+” signs and negative changes (ranking or rating decline) in red with " $\downarrow$ ” and "_“ signs. The zero changes of ranks and ratings (strictly said, their constituents - h3-index, citation count, and publication count) that did not change from the previous rating year are not explicitly displayed, however. The table is well arranged to see quickly that, for example, Philip A. Bernstein (rank 14, rating $22_{25}$ ) has dropped by 11 places since last year by decreasing his h3index from 6 to 2, receiving 63 citations less and publishing 2 papers less than in the 2011 rating. On the other hand, García-Molina (rank 1, rating $6_{167}$ ) maintained his rank but somewhat decreased his rating by keeping his h3-index and losing some citations and publications. Besides their names, the researchers in the table are indicated by their Scopus Author ID so that they can be identified unambiguously within Scopus. Of course, this rating table (Table 2) includes a very small number of researchers for whom the ratings could be computed manually. If researchers from a whole scientific field should be rated, the annual rating table would have to be generated automatically by means of computer programs. Since Scopus or Web of Science have data and software to produce world-wide scientometric indicators, they could easily integrate such annual field-specific rating tables of researchers within their products as a built-in feature. In fact, even researchers from various fields of science could be rated together if the underlying scientometric indicators (h-index, citation count, and publication number) and the time window length are corrected for the differences in publication and citation practices in those fields.

This is a preprint of an article accepted for publication in Journal of the American Society for Information Science and Technology copyright (C) 2013 (American Society for Information Science and Technology). 
TABLE 2. 2012 Current Index ratings of all Codd Award winners.

\begin{tabular}{|c|c|c|c|c|c|c|c|c|c|c|}
\hline Rank & Chg. & Name & Scopus ID & Rating & h3-index & Chg. & Citations & Chg. & Publications & Chg. \\
\hline 1 & & García-Molina, Héctor & 7005594983 & $6_{167}$ & 6 & & 167 & -29 & 30 & -5 \\
\hline 2 & $\uparrow 2$ & Chaudhuri, Surajit & 7402978010 & $5_{58}$ & 5 & & 58 & -9 & 26 & -1 \\
\hline 3 & $\downarrow 1$ & Stonebraker, Michael R. & 7005476233 & $4_{157}$ & 4 & -2 & 157 & +57 & 14 & -1 \\
\hline 4 & $\uparrow 1$ & Agrawal, Rakesh & 7201475122 & $4_{99}$ & 4 & & 99 & +23 & 18 & +7 \\
\hline 5 & $\uparrow 7$ & Dayal, Umeshwar & 7006545572 & $4_{63}$ & 4 & +1 & 63 & +38 & 48 & +5 \\
\hline 6 & $\uparrow 3$ & Vardi, Moshe Y. & 7005334525 & $4_{60}$ & 4 & & 60 & +20 & 36 & +3 \\
\hline 7 & & Dewitt, David J. & 7101912578 & $3_{125}$ & 3 & -1 & 125 & +61 & 5 & -1 \\
\hline 8 & $\downarrow 2$ & Widom, Jennifer & 7006676535 & $3_{51}$ & 3 & -1 & 51 & -13 & 11 & +3 \\
\hline 9 & $\downarrow 1$ & Abiteboul, Serge & 7005292791 & $3_{38}$ & 3 & -1 & 38 & -12 & 21 & +3 \\
\hline 10 & & Fagin, Ronald & 7005757964 & $3_{32}$ & 3 & -1 & 32 & & 14 & +1 \\
\hline 11 & $\uparrow 2$ & Carey, Michael J. & 7202744401 & $3_{29}$ & 3 & +1 & 29 & +2 & 12 & +2 \\
\hline 12 & $\downarrow 1$ & Gray, Jim O M & 7404300349 & $2_{69}$ & 2 & -1 & 69 & -392 & 2 & -3 \\
\hline 13 & $\uparrow 2$ & Kitsuregawa, Masaru & 7005566641 & $2_{34}$ & 2 & +1 & 34 & +19 & 47 & -4 \\
\hline 14 & $\downarrow 11$ & Bernstein, Philip A. & 7102505937 & 225 & 2 & -4 & 25 & -63 & 16 & -2 \\
\hline 15 & $\uparrow 1$ & Ullman, Jeffrey D. & 7004490091 & 2 & 2 & +1 & 14 & +13 & 7 & +3 \\
\hline 16 & $\downarrow 2$ & Maier, David & 7103065333 & 19 & 1 & -1 & 9 & & 11 & -2 \\
\hline 17 & & Selinger, Patricia Griffiths & 6701317222 & $1_{2}$ & 1 & +1 & 2 & +2 & 3 & +2 \\
\hline 18 & $\uparrow 1$ & Mohan, Chander $\mathrm{K} \mathrm{J}$ & 7102973829 & $0_{0}$ & 0 & & 0 & & 2 & +2 \\
\hline 19 & & Chamberlin, Donald D. & 7005587366 & $0_{0}$ & 0 & & 0 & & 0 & \\
\hline 19 & $\downarrow 2$ & Bayer, Rudolf & 7201391304 & $0_{0}$ & 0 & & 0 & & 0 & -1 \\
\hline
\end{tabular}

This is a preprint of an article accepted for publication in Journal of the American Society for Information Science and Technology copyright (C) 2013 (American Society for Information Science and Technology). 
The 3-year time window appears reasonable in the database field (and probably also in many other fields), but it can be adjusted to get a good balance between currency and sufficiency of publication/citation information in the research disciplines, where it is necessary. The time window length also influences how fast non-active researchers obtain a zero rating. With a 3year time window, two consecutive years of inactivity can still yield a non-zero rating that grows or declines from the previous year. Alternatively to the rating progress charts in Figure 1, where the actual ratings can be seen, also ranking progress charts with researchers' ranks might be presented. This is commonplace in tennis, where the actual ratings are much less important than players' ranks. However, we believe that a researcher's current scientific performance is better reflected by a rating (rather than a rank), similarly to chess.

One might argue that if the publication and citation windows are the same (they are in a complete overlay), publications near the end of the publication (and citation) window have less time to collect citations than publications from the beginning of the time window. This is certainly true, but if that property is the same for all researchers in a scientific field, it may still be fair to compare the scientists using the same (3-year) publication/citation window. Alternatively, we propose two other time windows and present the ratings of García-Molina based on them in Table 3. The first variant is a 2-year publication window (rating year minus 4 and rating year minus 3) and a 4-year citation window (the four years preceding the rating year). For example, in 2003 the publication window is 1999 and 2000 and the citation window is the period 1999 - 2002. In this case, papers published in 2000 have a shorter citation window than those published in 1999, but all papers have two years at least (2001 and 2002) to gather citations (denoted as h4-index in Table 3). In the second alternative, the time window is defined as above, but there is a sliding 3-year citation window, e.g. in 2003 we look at publications from 1999 and their citations in $1999-2001$ and at publications from 2000 and their citations in $2000-2002$ (denoted as h4'-index in Table 3). Using this definition, all publications have "equal" conditions to obtain citations. (Of course, it can still happen that a paper published in January has a longer citation window than another paper published in December of the same year.)

One of the reviewers argued that there was a problem with the Current Index ignoring citations occurring within the time window to papers outside of (i.e. prior to) the time window and suggested that also "current" citations to "earlier" papers should contribute to the index. This is actually represented by a fixed 3-year citation window and a floating (ever-growing) publication window. While it is true that reflecting earlier work may bring more justice to the

This is a preprint of an article accepted for publication in Journal of the American Society for Information Science and Technology copyright (C) 2013 (American Society for Information Science and Technology). 
rating and that inactive researchers may still have a non-zero rating with this approach, another problem arises: the rating loses its dynamics. This is demonstrated by the last five columns of Table 3 and denoted as h3'-index. As the numbers of publications used to calculate the index form a non-decreasing series by definition, the h3'-index will typically decline quite rarely or not decline at all as we can see with García-Molina whose rank remains static as well. In fact, 9 of the 20 researchers (45\%) under study never experienced a decrease of their h3'-index compared to only 1 out of 20 (5\%) whose $C I$ never declined. Also, only $14 \%$ of all changes in the h3'-index were decreases whereas $34 \%$ of all changes in the $C I$ were declines giving the rating equal chances to grow, fall, or stagnate. Moreover, the different natures of the h3'-index and CI are documented with quite uncorrelated rankings of scientists in various years with an average Pearson correlation coefficient of 0.286 . Therefore, h3'-index cannot be used as a dynamic rating system.

We computed Spearman's rank correlation coefficients between the ten Current Index rankings from 2003 to 2012 of twenty E. F. Codd Award winners in 1992 - 2011 and the respective rankings based on the other two definitions of the time window (h4-index and h4'index) and found a strong positive correlation ranging from 0.741 in 2009 to 0.971 in 2008 (with all coefficients being significant at the 0.01 level two-tailed). Regarding this high correlation and the simplicity and intuitive notion of the 3-year publication/citation window, it may be preferable to the other two time windows, especially with small-scale manual rating calculations, e.g. using the Scopus website. However, for automatic large-scale calculations based on off-line data, different publication/citation window definitions might also be considered. To show that Current Index works in other research disciplines as well, we computed ratings of twenty Priestley Medal ${ }^{4}$ recipients in 1992 - 2011 (awarded by the American Chemical Society) and present the annual rating table for 2012 in Table 4. Unlike the database researchers in Table 2, there is a greater number of zero-rated chemistry researchers in Table 4, which may indicate that the Priestley Medal is more often conferred to scientists who are at the end of their careers or even no longer active.

\footnotetext{
${ }^{4}$ Priestley Medal, http://webapps.acs.org/findawards/detail.jsp?ContentId=CTP_004545

This is a preprint of an article accepted for publication in Journal of the American Society for Information Science and Technology copyright (C) 2013 (American Society for Information Science and Technology).
} 
TABLE 3. Alternative ratings of Héctor García-Molina using different publication/citation windows.

\begin{tabular}{|c|c|c|c|c|c|c|c|c|c|c|c|c|c|c|c|}
\hline Year & Rating & h4 & Citations & Publications & Rank & Rating & h4 & Citations & Publications & Rank & Rating & h3' & Citations & Publications & Rank \\
\hline 2003 & $5_{67}$ & 5 & 67 & 19 & 1 & $4_{47}$ & 4 & 47 & 19 & 2 & $13_{554}$ & 13 & 554 & 161 & 1 \\
\hline 2004 & $4_{41}$ & 4 & 41 & 17 & 4 & $3_{36}$ & 3 & 36 & 17 & 3 & $14_{734}$ & 14 & 734 & 183 & 1 \\
\hline 2005 & $6_{101}$ & 6 & 101 & 20 & 1 & $5_{94}$ & 5 & 94 & 20 & 1 & $16_{969}$ & 16 & 969 & 200 & 1 \\
\hline 2006 & $10_{290}$ & 10 & 290 & 34 & 1 & $9_{204}$ & 9 & 204 & 34 & 1 & $19_{1269}$ & 19 & 1269 & 215 & 1 \\
\hline 2007 & $9_{271}$ & 9 & 271 & 39 & 1 & $7_{182}$ & 7 & 182 & 39 & 1 & $21_{1541}$ & 21 & 1541 & 223 & 1 \\
\hline 2008 & $7_{152}$ & 7 & 152 & 31 & 3 & 5105 & 5 & 105 & 31 & 4 & $22_{1763}$ & 22 & 1763 & 229 & 1 \\
\hline 2009 & $6_{132}$ & 6 & 132 & 22 & 7 & $5_{105}$ & 5 & 105 & 22 & 6 & $23_{1929}$ & 23 & 1929 & 242 & 1 \\
\hline 2010 & $4_{123}$ & 4 & 123 & 14 & 7 & $4_{94}$ & 4 & 94 & 14 & 5 & $24_{2041}$ & 24 & 2041 & 257 & 1 \\
\hline 2011 & $5_{179}$ & 5 & 179 & 19 & 4 & $5_{155}$ & 5 & 155 & 19 & 3 & $25_{2196}$ & 25 & 2196 & 264 & 1 \\
\hline 2012 & $9_{343}$ & 9 & 343 & 28 & 1 & $8_{262}$ & 8 & 262 & 28 & 1 & $27_{2306}$ & 27 & 2306 & 273 & 1 \\
\hline
\end{tabular}

TABLE 4. 2012 Current Index ratings of 20 Priestley Medal winners.

\begin{tabular}{|c|c|c|c|c|c|c|c|c|c|c|}
\hline Rank & Chg. & Name & Scopus ID & Rating & h3-index & Chg. & Citations & Chg. & Publications & Chg. \\
\hline 1 & & Whitesides, George M. & 36038822100 & $17_{890}$ & 17 & -1 & 890 & -107 & 105 & \\
\hline 2 & & Somorjai, Gábor A. & 35396886300 & $14_{682}$ & 14 & & 682 & -155 & 67 & -10 \\
\hline 3 & $\uparrow 1$ & Bard, Allen J. & 35350527400 & $12_{371}$ & 12 & & 371 & -30 & 56 & -2 \\
\hline 4 & $\downarrow 1$ & Zewail, Ahmed H. & 7004914740 & $11_{394}$ & 11 & -1 & 394 & -17 & 53 & -3 \\
\hline 5 & $\uparrow 2$ & Oláh, George Andrew & 36045924000 & $7_{232}$ & 7 & & 232 & +99 & 48 & +8 \\
\hline 6 & $\downarrow 1$ & Corey, Elias James & 7202254852 & $7_{163}$ & 7 & -2 & 163 & -29 & 21 & -11 \\
\hline 7 & $\downarrow 1$ & Zare, Richard N. & 35355951800 & $7_{151}$ & 7 & & 151 & -38 & 46 & +5 \\
\hline 8 & & Breslow, Ronald C. & 24443481400 & $7_{143}$ & 7 & +2 & 143 & +64 & 20 & +5 \\
\hline 9 & $\uparrow 1$ & Frederick Hawthorne, M. Frederick & 7102788963 & $3_{23}$ & 3 & & 23 & +11 & 17 & +3 \\
\hline 10 & $\uparrow 1$ & Hoffman, Darleane C. & 7402222195 & $3_{10}$ & 3 & +1 & 10 & -2 & 4 & -2 \\
\hline 11 & $\downarrow 2$ & Albert Cotton, F. Albert & 7201778183 & $2_{14}$ & 2 & -2 & 14 & -26 & 9 & -5 \\
\hline 12 & & Djerassi, Carl & 35600002000 & $1_{1}$ & 1 & +1 & 1 & +1 & 2 & -1 \\
\hline 13 & & Anderson, Paul S. & 7404425321 & $\mathrm{O}_{0}$ & 0 & & 0 & & 0 & \\
\hline 14 & & Barton, Derek HR R & 9272689500 & $\mathrm{O}_{0}$ & 0 & & 0 & & 0 & \\
\hline 15 & & Basolo, Fred & 7007150726 & $0_{0}$ & 0 & & 0 & & 0 & \\
\hline 16 & & Eliel, Ernest L. & 7004876653 & $0_{0}$ & 0 & & 0 & & 0 & \\
\hline 17 & & Good, Mary L. & 7202187884 & $0_{0}$ & 0 & & 0 & & 0 & \\
\hline 18 & & Parry, Robert W. & 7101830447 & $0_{0}$ & 0 & & 0 & & 0 & \\
\hline 19 & & Simmons, Howard E. & 35576856300 & $\mathrm{O}_{0}$ & 0 & & 0 & & 0 & \\
\hline 19 & & Vandenberg, Edwin J. & 7003531370 & $0_{0}$ & 0 & & 0 & & 0 & \\
\hline
\end{tabular}

This is a preprint of an article accepted for publication in Journal of the American Society for Information Science and Technology copyright (C) 2013 (American Society for Information Science and Technology). 


\section{Concluding remarks}

Stefani (2011) reports that out of 159 international sports federations under study, only 60 (or $38 \%$ ) have no rating system at all. On the other hand, 84 sports federations (53\%) use an accumulative rating system, in which points are gathered over a time window. In these systems, senior and junior players have the same starting positions, which allows for an immediate comparison of players of different seniority. This stands in a stark contrast to the current scientometric indicators of the performance of researchers such as h-index or citation counts that accrue non-decreasingly over the researchers' careers and, therefore, are biased towards more senior scientists. To overcome this problem, we have introduced the Current Index which is an h-index based on a 3-year publication/citation window combined with a citation count for that time period. If the Current Index is equal for two or more researchers, the number of papers published in the specific period is used as a tiebreak criterion. We have shown that if the rating is updated regularly (possibly on a yearly basis), it may present a dynamic rating framework in which researchers' ratings (and ranks) can grow as well as decline in time according to their most recent performance like in many sports rating systems. A researcher's Current Index can be presented as a single (compound) number and its development is easy to visualize by means of a rating progress chart. Although we demonstrated the rating system on a very small set of researchers (ACM SIGMOD Edgar F. Codd Innovations Award winners and Priestley Medal recipients), it may be used to rate researchers in a whole scientific field or even across various fields if appropriate correction measures, which reflect different publication and citation patterns in those fields, are taken. However, these large-scale ratings cannot be performed manually, but the annual rating tables could be easily integrated within Scopus or Web of Science as a built-in feature. In addition, as athletes' life span and peak time can be very different from those of scientists, more evidence is needed before using the proposed mechanism to truly rate researchers.

\section{Acknowledgements}

This work was supported by the European Regional Development Fund (ERDF), project "NTIS - New Technologies for Information Society", European Centre of Excellence, CZ.1.05/1.1.00/02.0090. Many thanks are due to the reviewers for their insightful comments.

This is a preprint of an article accepted for publication in Journal of the American Society for Information Science and Technology copyright (C) 2013 (American Society for Information Science and Technology). 


\section{References}

Hirsch, J. E. (2005). An index to quantify an individual's scientific research output.

Proceedings of the National Academy of Sciences of the United States of America, 102(46), 16569-16572.

Hirsch, J. E. (2010). An index to quantify an individual's scientific research output that takes into account the effect of multiple coauthorship. Scientometrics, 85(3), 741-754.

Stefani, R. (2011). The methodology of officially recognized international sports rating systems. Journal of Quantitative Analysis in Sports, 7(4), art. no. 10. 